\title{
MicroRNA-625-3p improved proliferation and involved chemotherapy resistance via targeting PTEN in high grade ovarian serous carcinoma
}

\author{
Lili Zhong ${ }^{1}$, Xiumin Liu², Lixing Wang ${ }^{3}$, Yu Liư ${ }^{3}$, Duohan Zhang ${ }^{3}$ and Yinlong Zhao ${ }^{3^{*}}$
}

\begin{abstract}
Objective: High-grade serous ovarian cancer (HGSOC) is an aggressive gynaecological malignancy and associated with poor prognosis. Here we examined the effects of miR-625-3p on proliferation, treatment, migration and invasion in HGSOC.

Methods: The proliferation of HGSOC cells was evaluated by MTT assay. Transwell assay was performed to examine migration and matrigel assay were used to assess invasion. The effect of miR-625-3p on cisplatin-induced apoptosis was investigated by Caspase-Glo3/7 assay. The dual-luciferase reporter assay was carried out to confirm the potential binding site.

Results: Overexpression of miR-625-3p promoted proliferation, and increased migration and invasion in HGSOC cells. MiR-625-3p significantly inhibited cisplatin sensitivity in HGSOC cells. Meanwhile, miR-625-3p decreased cisplatininduced apoptosis by regulation of BAX and Bcl-2 expression. Furthermore, aberrant expression of miR-625-3p changed PTEN expression by directly binding to 3'UTR of PTEN. Further study showed miR-625-3p expression was higher in human HGSOC tissue than normal ovarian tissues and associated with higher clinical stage.

Conclusions: miR-625-3p promotes HGSOC growth, involves chemotherapy resistance and might serve as a potential biomarker to predict chemotherapy response and prognosis in HGSOC.
\end{abstract}

Keywords: miR-625-3p, HGSOC, Apoptosis, PTEN

\section{Introduction}

HGSOC is consisted of $60-80 \%$ of ovarian epithelial carcinoma, and patients with HGSOC most often present at advanced clinical stage and have poor outcome [1]. The first-line treatment of HGSOC is debulking surgery followed by chemotherapy [2]. Cisplatin interferes cell division and inhibits cancer cell proliferation, and has been used as a chemotherapy reagent for a variety of cancers including HGSOC [3]. However, studies have shown that cancer cells develop cisplatin resistance by reducing accumulation $[4,5]$. Patients who develop cisplatin resistance often have shorter survival time. Therefore, it is critical to find out the molecular mechanisms of cisplatin resistance and develop effective treatments in patients with HGSOC.

\footnotetext{
*Correspondence: yinlongzhao@aol.com

${ }^{3}$ Department of Nuclear Medicine, The Second Hospital of Jilin University,

No. 218, Ziqiang Street, Nanguan, Changchun 130041, Jilin, People's

Republic of China

Full list of author information is available at the end of the article
}

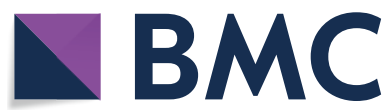

(c) The Author(s) 2022. Open Access This article is licensed under a Creative Commons Attribution 4.0 International License, which permits use, sharing, adaptation, distribution and reproduction in any medium or format, as long as you give appropriate credit to the original author(s) and the source, provide a link to the Creative Commons licence, and indicate if changes were made. The images or other third party material in this article are included in the article's Creative Commons licence, unless indicated otherwise in a credit line to the material. If material is not included in the article's Creative Commons licence and your intended use is not permitted by statutory regulation or exceeds the permitted use, you will need to obtain permission directly from the copyright holder. To view a copy of this licence, visit http://creativecommons.org/licenses/by/4.0/. The Creative Commons Public Domain Dedication waiver (http://creativeco mmons.org/publicdomain/zero/1.0/) applies to the data made available in this article, unless otherwise stated in a credit line to the data. 
Recently, growing evidence has shown that microRNAs impact essential cellular processes such as differentiation, proliferation and apoptosis in organ development and tumorigenesis by directly binding to 3'-untranslated region of target mRNAs [6, 7]. MiRNA profiling studies have shown aberrant miRNA expression in a variety of neoplasms including ovarian cancer [8]. Yang et al. showed that miRNA-802 inhibited growth and migration in ovarian cancer by regulating YWHAZ [9]. Rao et al reported that miR-195-5p suppressed ovarian cancer cell proliferation by directly targeting 3'-UTR of MICU1 mRNA and repressing MICU1 expression [10]. Xing et al found that miR-598 inhibited ovarian cancer cell growth and metastasis by regulating unconventional prefoldin RPB5 interactor, a member of the prefoldin family of molecular chaperones [11]. Interestingly, some studies have demonstrated that circulating miRNAs can be potential diagnostic biomarkers in patients with ovarian cancer $[12,13]$.
Table 1 The antibodies used in western blot

\begin{tabular}{lll}
\hline Antibody & Vendor & Dilution \\
\hline BAX & Cell signaling Technology, USA & $1: 1000$ \\
BCL-2 & Santa cruz Biotechnology, USA & $1: 1000$ \\
P-AKT & Cell signaling Technology, USA & $1: 500$ \\
PTEN & Santa cruz Biotechnology, USA & $1: 500$ \\
AKT & Cell signaling Technology, USA & $1: 1500$ \\
GAPDH & Santa cruz Biotechnology, USA & $1: 2000$ \\
\hline
\end{tabular}

In this study, we evaluated the effects of miR-625-3p in growth, cisplatin sensitivity, migration and invasion using HGSOC cells and tissue.

\section{Materials and methods \\ Ovarian cancer cell lines}

Two HGSOC cell lines, OVCAR3 and OVCAR4, were purchased from the American Type Culture Collection (ATCC, USA) and grown in Dulbecco's Modified Eagle's

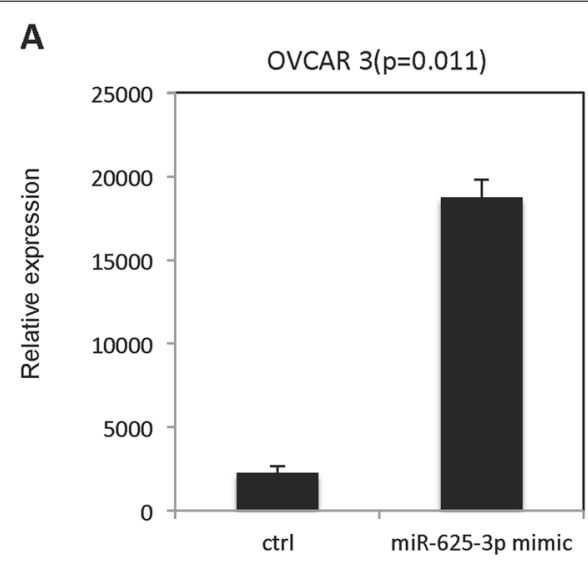

B

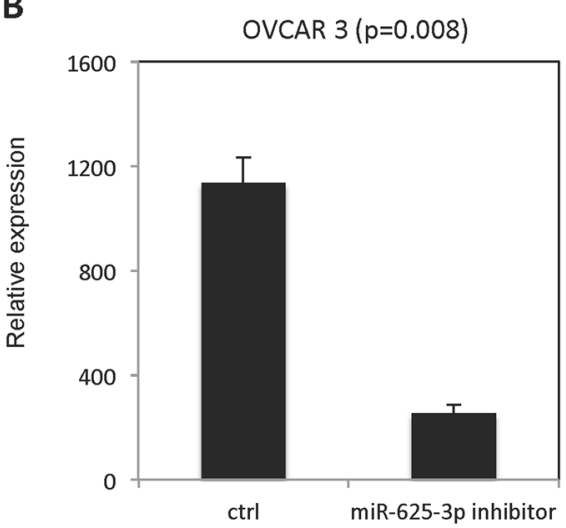

C

D
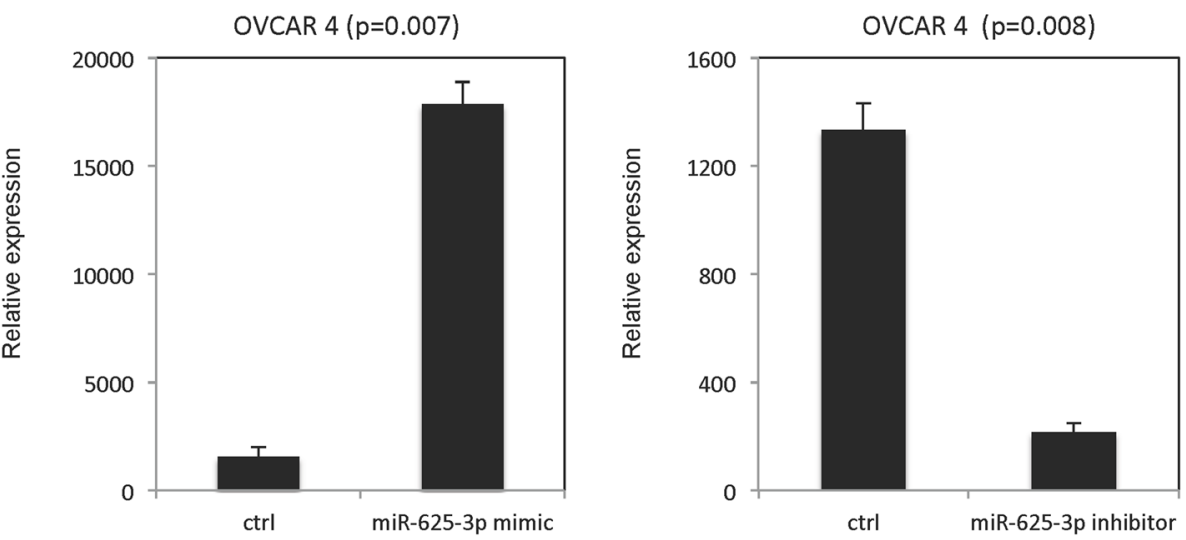

Fig. 1 miR-625-3p expression in OVCAR3 and OVCAR4 cell after transfected either with mimic or inhibitor. A miR-625-3p expression in OVCAR3 cells transfected with miR-625-3p mimic. B miR-625-3p expression in OVCAR3 cells transfected with miR-625-3p inhibitor. C miR-625-3p expression in OVCAR4 cells transfected with miR-625-3p mimic. D miR-625-3p expression in OVCAR4 cells transfected with miR-625-3p inhibitor 
Medium (DMEM) supplemented with 10\% fetal bovine serum, 100 units of penicillin/ml and $100 \mathrm{mg}$ of streptomycin/ml (Invitrogen, USA).

\section{MicroRNA transfection}

The miR-625-3p mimic and miR-625-3p inhibitor (Invitrogen, USA) were transfected to ovarian cancer cells using Lipofectamine 2000 (Invitrogen, USA). The scramble microRNA was used as negative control (Invitrogen, USA).

\section{Quantitative real-time polymerase chain reaction (qRT-PCR)}

Total RNA was extracted from ovarian cancer cells using TRIzol reagent (Invitrogen, USA). Quantitative Real-Time PCR (qRT-PCR) was performed to detect
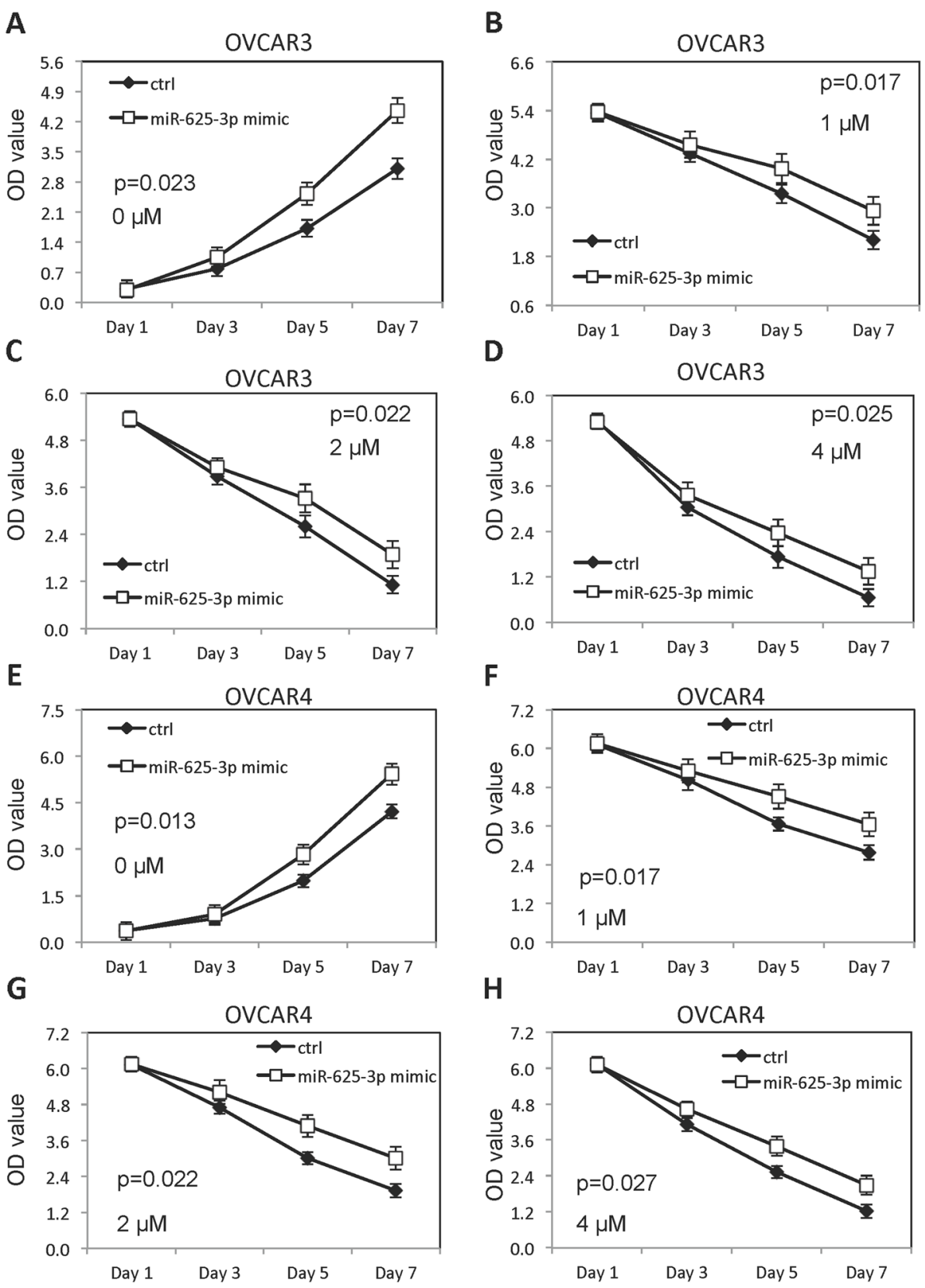

Fig. 2 Forced overexpression of miR-625-3p increased growth and inhibited cisplatin sensitivity. A Growth of OVCAR3 cells forced overexpressed miR-625-3p without cisplatin. B-D Growth of OVCAR3 cells forced overexpressed miR-625-3p in presence of different doses of cisplatin (1,2 and $4 \mu \mathrm{M})$. E Growth of OVCAR4 cells forced overexpressed miR-625-3p without cisplatin. F-H Growth of OVCAR4 cells forced overexpressed miR-625-3p in presence of different doses of cisplatin $(1,2$ and $4 \mu \mathrm{M})$ 
miR-625-3p expression level. The reactions were performed as follows: $94^{\circ} \mathrm{C}$ for $1 \mathrm{~min}, 36$ cycles at $94^{\circ} \mathrm{C}$ for $30 \mathrm{~s}, 60^{\circ} \mathrm{C}$ for $25 \mathrm{~s}$, and a dissociation stage. The primers were synthesized by Sigma (USA) and the sequences were as follow: miR-625-3p forward: 5' ATTAGATTG CTAACTAGC-3' and reverse 5'- TTAGTACGAATT ATCGTAA-3. U6 was included as endogenous control.

\section{Cell proliferation assay}

MTT assay was used to evaluate ovarian cancer cell proliferation. Briefly, OVCAR3 and OVCAR4 cells were seeded in 96-well plates and cultured overnight. Then different concentration of cisplatin $(0,2,4$ and $6 \mu \mathrm{M})$ was added. Every 48 hours, MTT reagents was added and incubated for 4 hours. Then DMSO was added to dissolve the formed
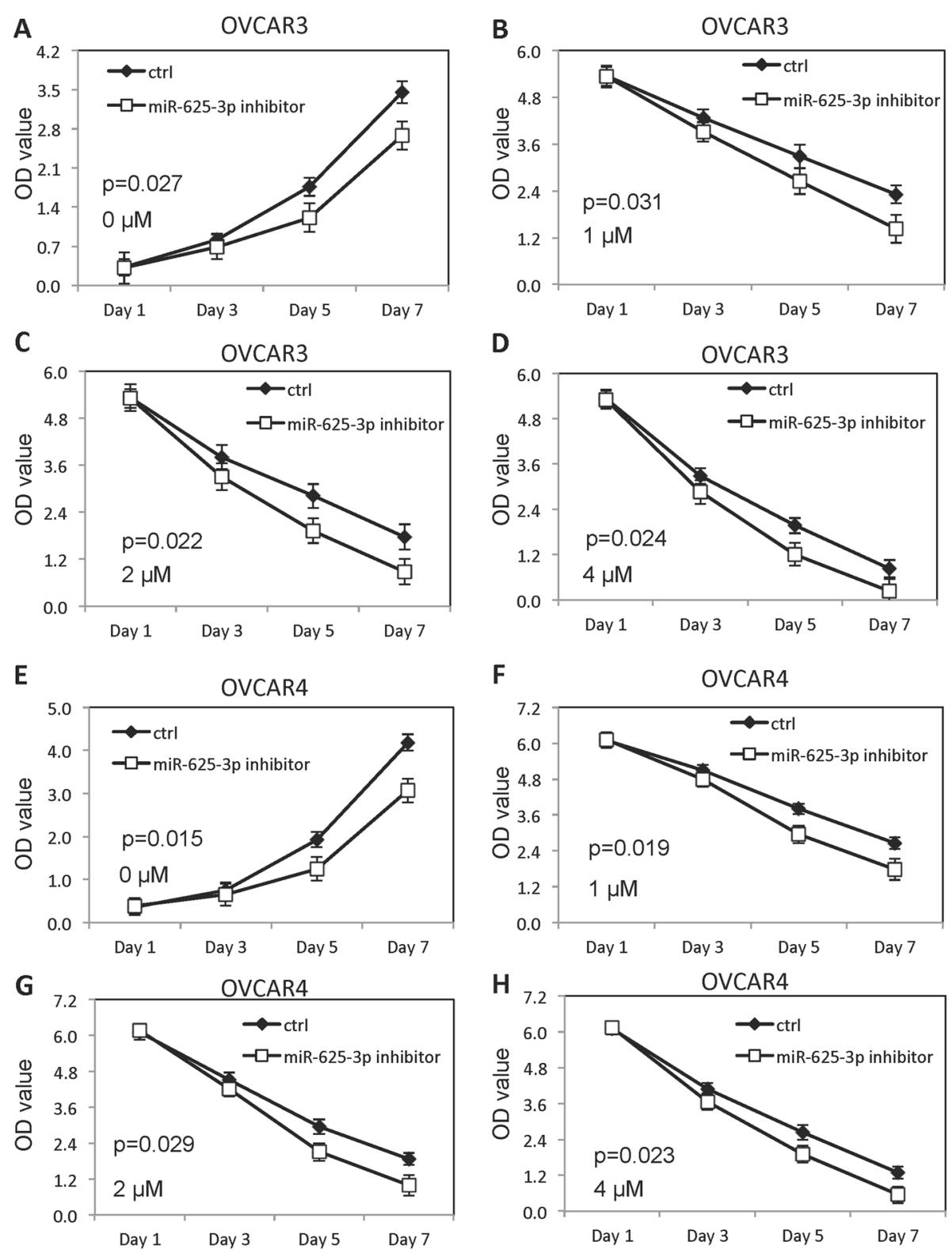

Fig. 3 Low level of miR-625-3p decreased growth and promoted cisplatin sensitivity. A Growth of OVCAR3 cells decreased miR-625-3p without

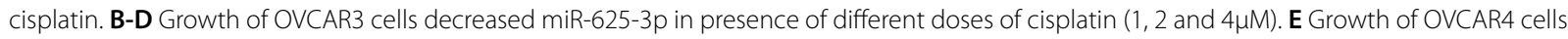
decreased miR-625-3p without cisplatin. F-H Growth of OVCAR4 cells decreased miR-625-3p in presence of different doses of cisplatin (1, 2 and $4 \mu \mathrm{M})$ 
A

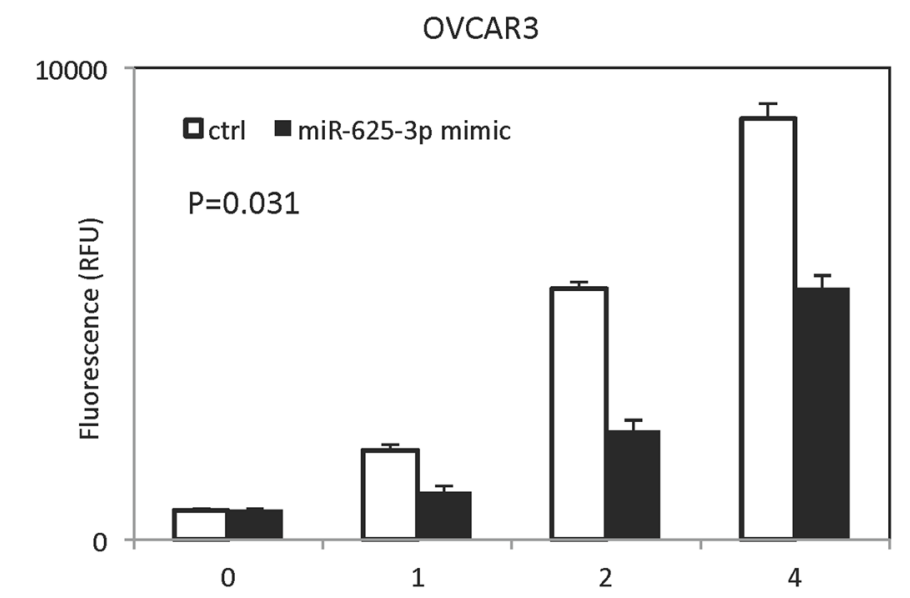

B

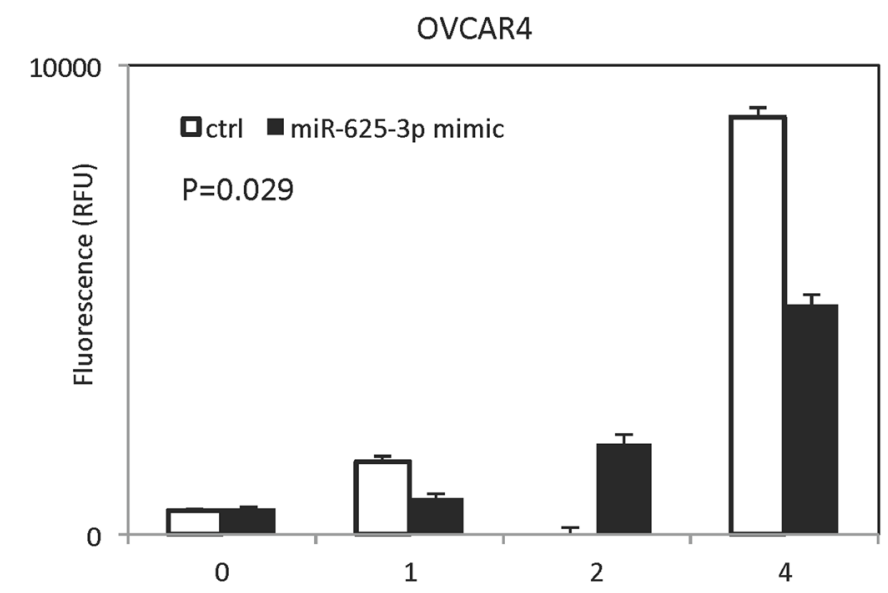

C
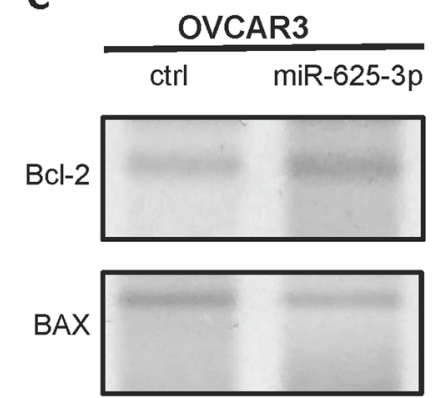

GAPDH

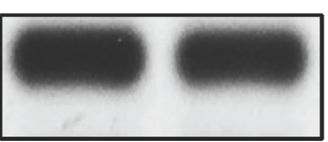

D
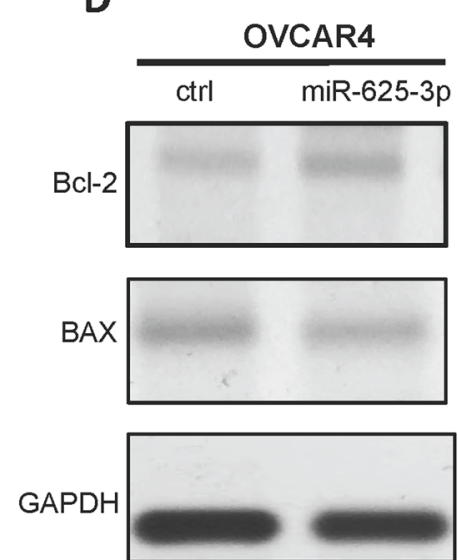

Fig. 4 miR-625-3p inhibits cisplatin-induced apoptosis. A miR-625-3p decreased caspase 3/7 activity in OVCAR3 cells treated with cisplatin in dose dependent manner. B miR-625-3p decreased caspase 3/7 activity in OVCAR4 cells treated with cisplatin in dose dependent manner. C-D miR-625-3p regulates BAX and BCL-2 expression in OVCAR3 and OVCAR4 cells treated with cisplatin 
crystal. The signal was detected on a microplate reader (Invitrogen, USA) at $570 \mathrm{~nm}$.

\section{Caspase $3 / 7$ apoptosis assay}

Ovarian cancer cells were incubated with different concentrations of cisplatin $(0,2,4$ and $6 \mu \mathrm{M})$ for 48 hours. Then Caspase-Glo reagent (Promega, USA) was added and incubated for 2 hours. Luminescence was evaluated with parameters of 1 minute lag time and 0.5 second/ well read time in a plate-reading luminometer (ThermoFisher, USA).

\section{Transwell assay}

The invasion assay was conducted using upper chambers coated with Matrigel (BD Bioscience, USA). The migration assay was performed using the top chambers without Matrigel. Briefly, ovarian cancer cells were placed in upper chambers in serum free culture medium, and
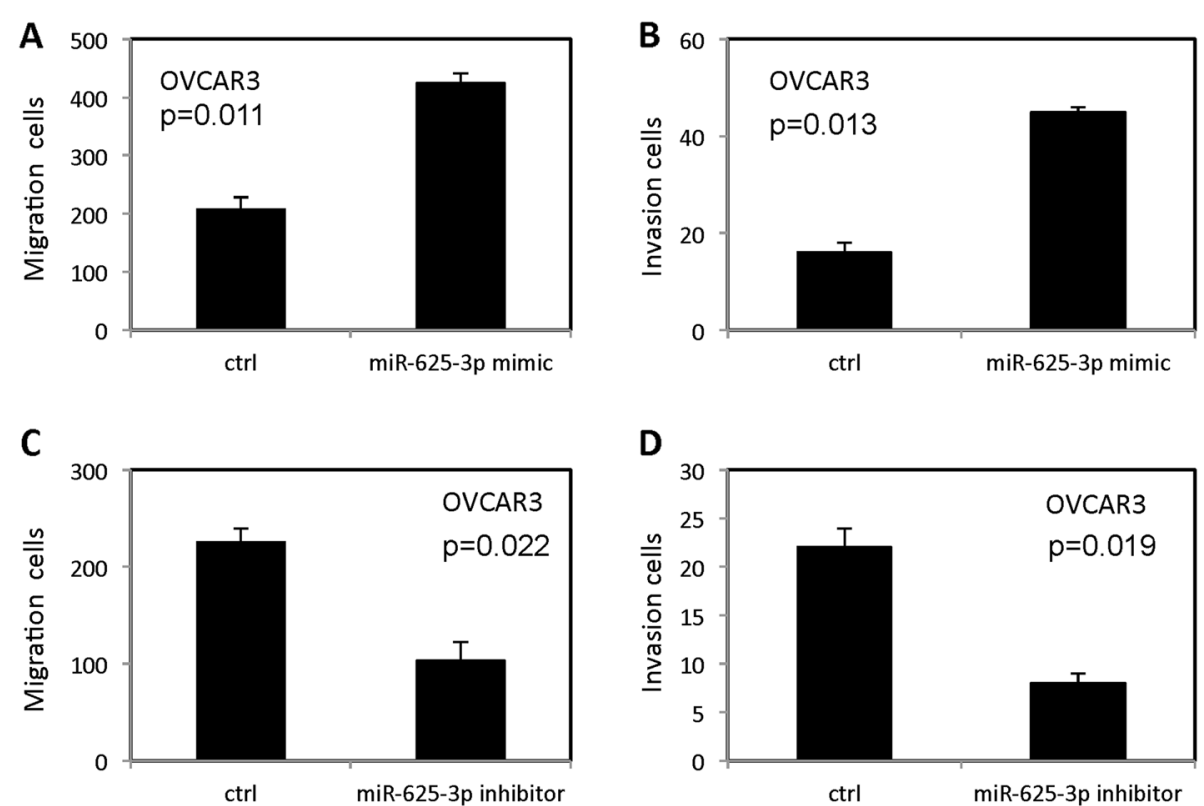

D
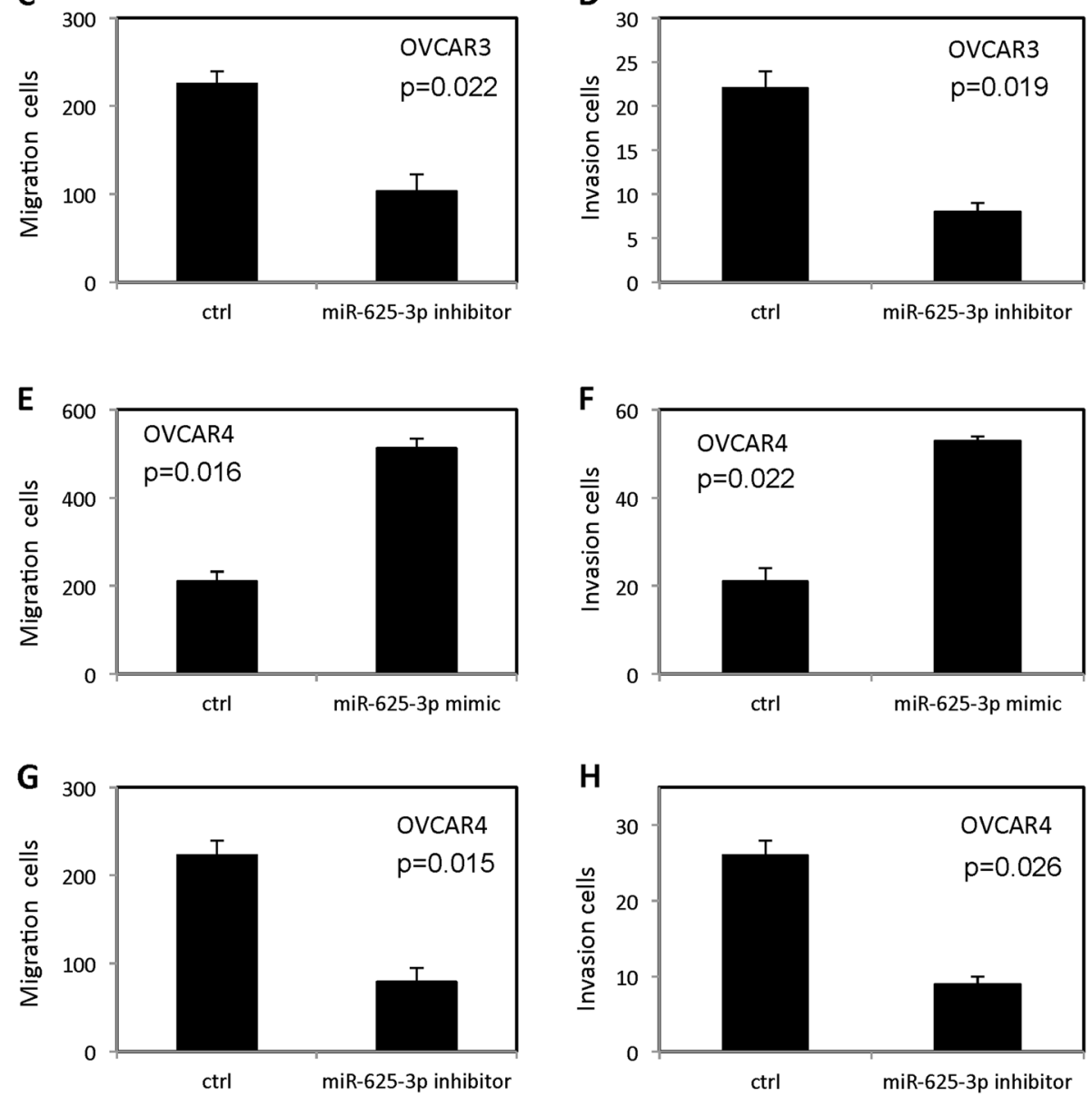

Fig. 5 miR-625-3p regulated invasion and migration in OVCAR3 and OVCAR4 cells. A-B Forced overexpression of miR-625-3p promoted migration and invasion in OVCAR3 cells. C-D Low level miR-625-3p inhibited migration and invasion in OVCAR3 cells. E-F Forced overexpression of miR-625-3p promoted migration and invasion in OVCAR4 cells. G-H Low level miR-625-3p inhibited migration and invasion in OVCAR4 cells 

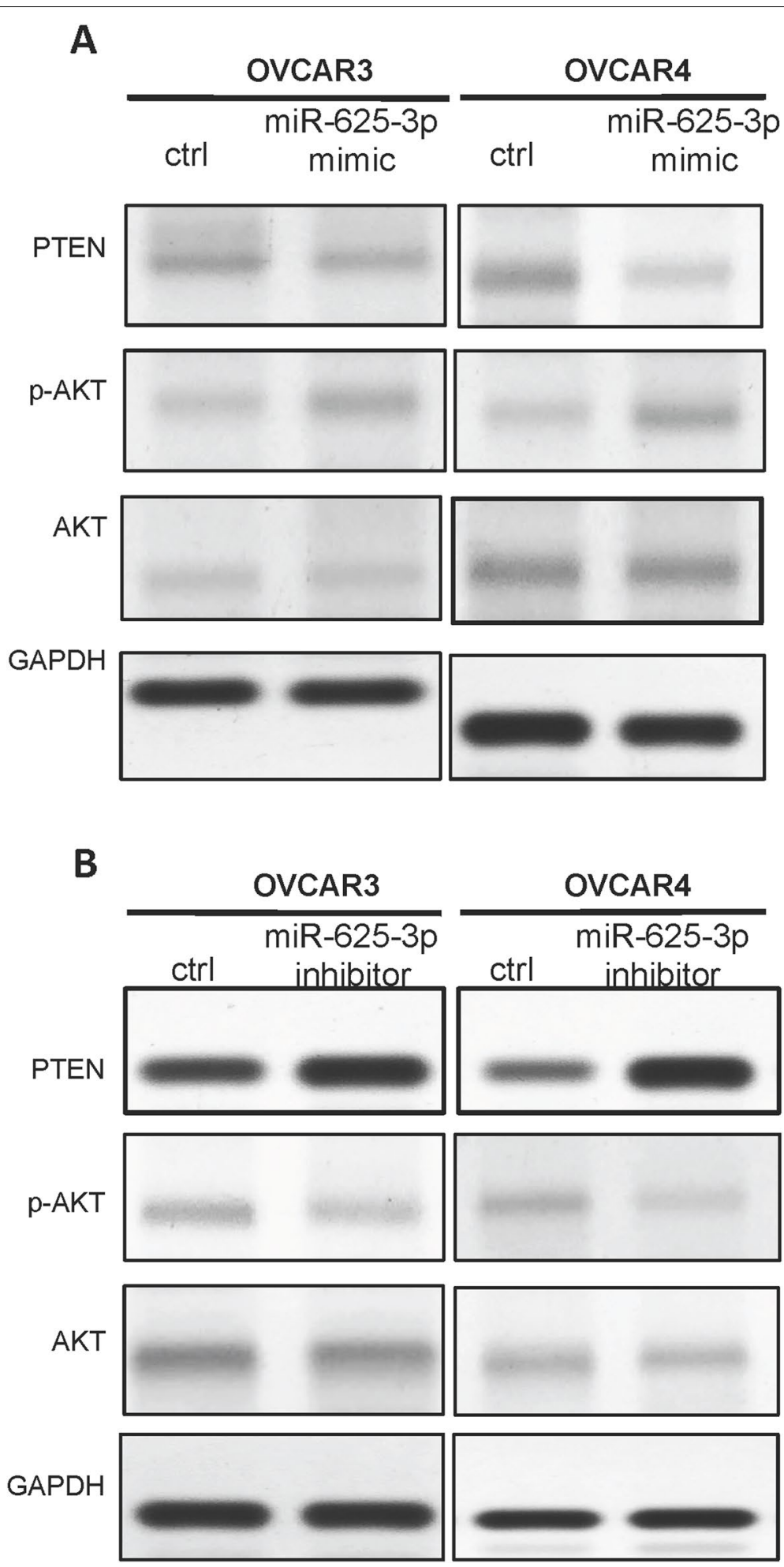

Fig. 6 miR-625-3p regulated PTEN/AKT protein expression. A Forced overexpression miR-625-3p regulated expression of p-AKT, AKT and PTEN in OVCAR3 and OVCAR4 cells. B Low level of miR-625-3p regulated expression of p-AKT, AKT and PTEN in OVCAR3 and OVCAR4 cells 
culture medium supplemented with 10\% FBS was added into lower chambers. After 18 hours culture, the invaded cells on the bottom of membrane were stained and counted at randomly fields.

\section{Western blotting}

Total protein was extracted from ovarian cancer cells and separated by SDS-PAGE gel electrophoresis. The proteins were transferred to PVDF membranes (Sigma, USA). After blocking with 5\% nonfat dried milk, the PVDF membranes were incubated with different primary antibodies (Table 1 ) overnight at $4^{\circ} \mathrm{C}$ with gentle agitation, followed by incubation with secondary antibodies. The signal on the PVDF membrane was examined by enhanced chemiluminescence reagent (Pierce, USA).

\section{Luciferase assay}

The potential binding sites of miR-625-3p were identified by checking miRDB online database. The sequence of PTEN 3'-UTR containing the putative miR-625-3p binding site was amplified from human normal cell genomic DNA. Using the In-Fusion Dry-Down PCR Cloning Kit (Clontech, USA), the amplified sequence was cloned into the Xbal site of the pmirGLO Dual-Luciferase miRNA

A

PTEN: 5'-.. CGAUGAGAUAGUGAGCGGUA...3'-

| | | || |

Has-miR-625-3p: 3'- .. AGCUGAUCUCAGCUCGCCUG-5'

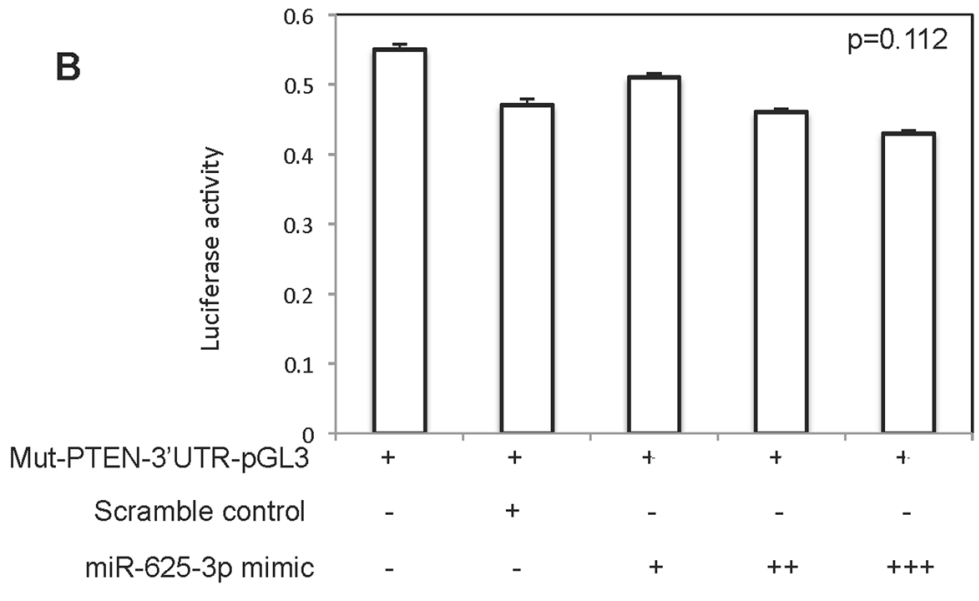

C
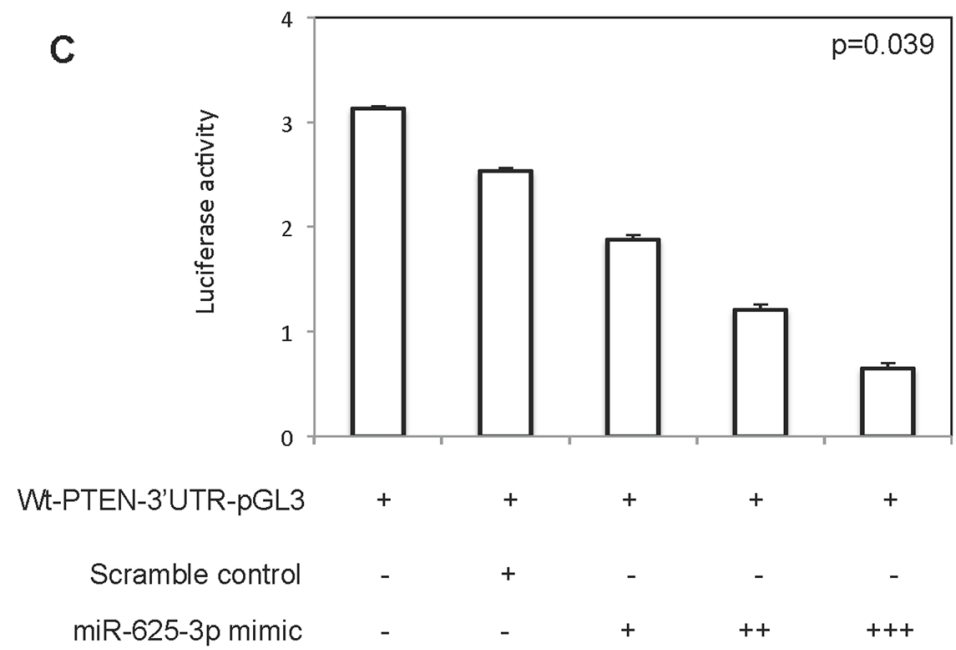

Fig. 7 miR-625-3p directly targeted PTEN. A The possible binding site of miR-625-3p to PTEN; B Luciferase activity of OVCAR3 cells co-transfected miR-625-3p mimic with mut-PTEN-3'-UTR-pGL3; C Luciferase activity of OVCAR3 cells co-transfected miR-625-3p mimic with wt-PTEN-3'-UTR-pGL3 
Target Expression Vector (Promega, USA). Three point mutations were introduced into the seed region of the miR-625-3p binding sites, which was used as control. OVCAR3 cells were co-transfected with Luc-PTEN and miR-625-3p using Lipofectamine RNAiMAX. After 48 hours, luciferase activities were measured using the Dual-Luciferase Reporter Assay system (Promega, USA).

\section{MiR-625-3p expression on human ovarian cancer tissue}

Formalin-fixed paraffin-embedded (FFPE) tissue was collected from 155 patients with ovarian cancer between 2005 and 2020. This study was approved by ethical committee of Jilin University. The informed consents were collected from every patient. The diagnosis was confirmed by two experienced pathologists and qRT-PCR were performed using total RNA extracted from FFPE tissue to evaluate miR-625-3p levels.

\section{Immunohistochemical analysis}

PTEN and Ki-67 immunostains were performed. The normal ovarian tissue adjacent to ovarian cancer tissue was used as control. Immunohistochemical stain was performed on automated stainer (Bond RX, USA) using PTEN (Cell Signaling, 1:1500) and Ki-67 (Cell Signaling, 1:1500) antibodies.

\section{Statistical analysis}

SPSS 12.0 statistical software (IBM Corp, USA) was used for statistical analysis. $\mathrm{P}<0.05$ was considered statistically significant using one-way analysis of variance (Tukey, ANOVA).

\section{Results}

MiR-625-3p increased growth of OVCAR3 and OVCAR4 and inhibited cisplatin sensitivity

miR-625-3p expression was dramatically increased in OVCAR3 (Fig. 1A) and OVCAR4 (Fig. 1C) cells after transfection of miR-625-3p mimic. However, miR-625-3p expression was significantly decreased in OVCAR3 (Fig. 1B) and OVCAR4 (Fig. 1D) cells after transfection of miR-625-3p inhibitor. Growth of OVCAR3 and OVCAR4 cells in absence and presence of cisplatin was checked by MTT assay. As shown in Fig. 2, forced overexpression of miR-625-3p improved growth of OVCAR3 cells (Fig. 2A) and OVCAR4 cells (Fig. 2E). Meanwhile, forced overexpression of miR-625-3p inhibited cisplatin sensitivity in a dose dependent manner both in OVCAR 3 cells (Fig. 2BD) and OVCAR4 cells (Fig. 2F-H). On the contrast, low expression of miR-625-3p decreased growth of OVCAR3 cells (Fig. 3A) and OVCAR4 cells (Fig. 3E). Meanwhile, low expression of miR-625-3p improved cisplatin sensitivity in a dose dependent manner both in OVCAR 3 cells (Fig. 3B-D) and OVCAR4 cells (Fig. 3F-H).

\section{MiR-625-3p decreased cisplatin-induced apoptosis in OVCAR3 and OVCAR4 cells}

Caspase $3 / 7$ activity was performed to examine the effect of miR-625-3p on cisplatin-induced apoptosis. Forced overexpression of miR-625-3p decreased cisplatin-induced apoptosis in OVCAR3 cells (Fig. 4A) and OVCAR4 cells (Fig. 4B). Meanwhile, high level of miR625-3p inhibited BAX and increased Bcl-2 in OVCAR3 cells and OVCAR4 cells (Fig.4C-D).

\section{MiR-625-3P promoted invasion and migration in OVCAR3 and OVCAR 4 cell}

As shown in Fig. 5, forced overexpression of miR-625-3p promoted migration (Fig. 5A and E) and invasion (Fig. 5B and F) in OVCAR3 and OVCAR4 cells. In contrast, low expression of miR-625-3p inhibited migration (Fig. 5C and $\mathrm{G}$ ) and invasion (Fig. 5D and $\mathrm{H}$ ) in OVCAR3 cells and OVCAR4 cells.

Table 2 miR-625-3p expression level and Ki-67 index in different stage high grade ovarian serous cancer

\begin{tabular}{|c|c|c|c|c|}
\hline Stage & Number of patients & $\begin{array}{l}\text { miR-625-3p levels in normal breast } \\
\text { tissue }\end{array}$ & $\begin{array}{l}\text { miR-625-3p levels in invasive cancer } \\
\text { tissue }\end{array}$ & $\begin{array}{l}\text { Ki-67 index } \\
\text { (per } 2 \\
\mathrm{~mm} 2 \text { ) }\end{array}$ \\
\hline $\mid A$ & 25 & $103 \pm 6$ & $221 \pm 33$ & $16 \pm 2$ \\
\hline $\mathrm{IB}$ & 28 & $122 \pm 11$ & $239 \pm 25$ & $17 \pm 1$ \\
\hline IC & 21 & $110 \pm 10$ & $223 \pm 29$ & $15 \pm 2$ \\
\hline$\| A$ & 21 & $101 \pm 11$ & $389 \pm 21$ & $22 \pm 2$ \\
\hline$\| \mathrm{B}$ & 18 & $93 \pm 6$ & $321 \pm 25$ & $26 \pm 4$ \\
\hline$\| \mathrm{A}$ & 14 & $101 \pm 9$ & $471 \pm 23$ & $35 \pm 4$ \\
\hline$\| I \mathrm{~B}$ & 12 & $117 \pm 7$ & $464 \pm 21$ & $37 \pm 2$ \\
\hline $\mathrm{IIIC}$ & 7 & $106 \pm 11$ & $485 \pm 26$ & $41 \pm 3$ \\
\hline IVA & 6 & $102 \pm 14$ & $536 \pm 17$ & $47 \pm 2$ \\
\hline IVB & 3 & $131 \pm 19$ & $544 \pm 19$ & $49 \pm 5$ \\
\hline
\end{tabular}



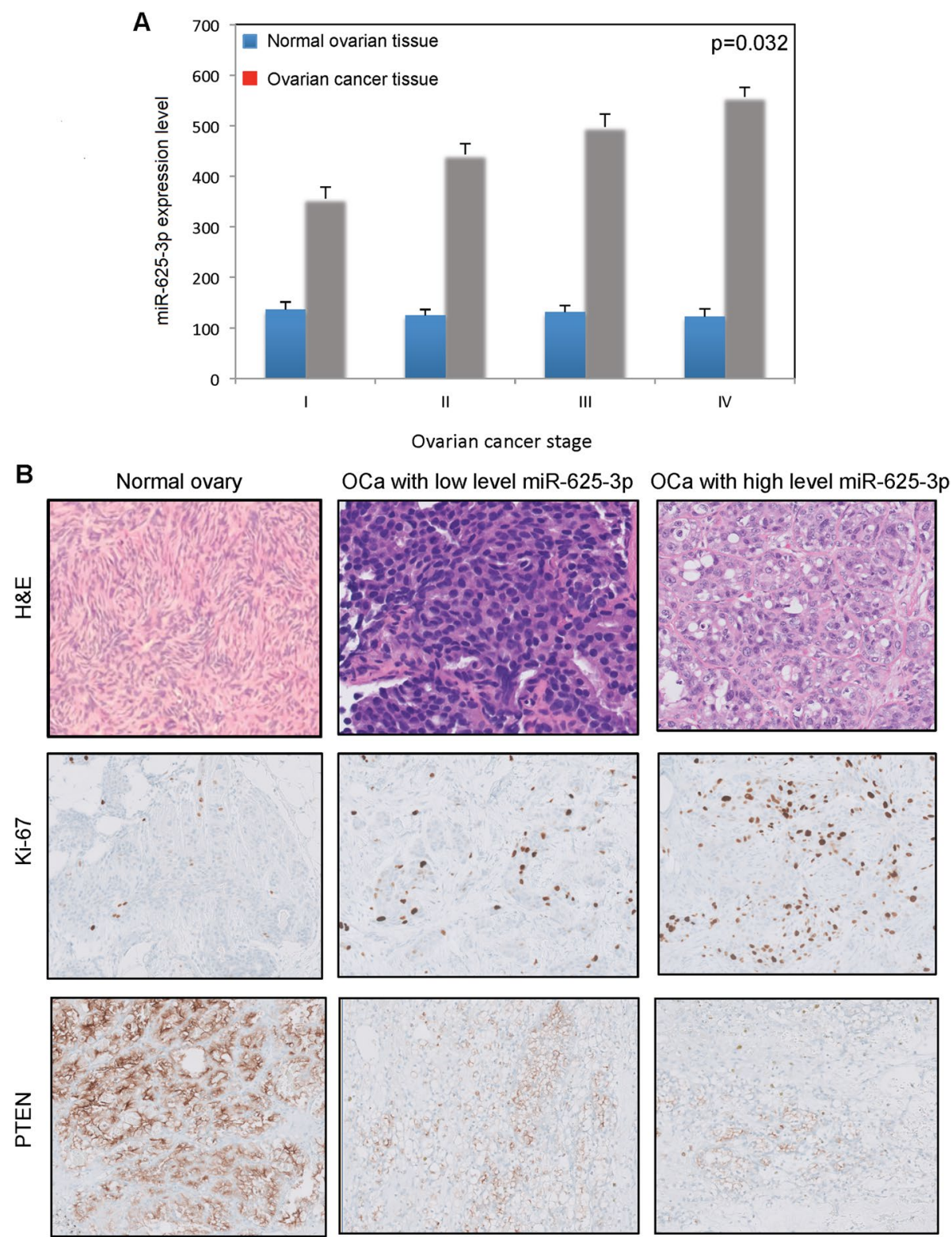

Fig. 8 miR-625-3p level in human high-grade serous carcinoma tissue. A miR-625-3p level in different stage ovarian cancer. B Immunohistochemical studies for Ki-67 and PTEN on normal ovarian tissue, ovarian cancer with low level miR-625-3p and ovarian cancer with high level miR-625-3p

\section{MiR-625-3p decreased PTEN expression in OVCAR3 and OVCAR4 cells by directly binding to 3 '-UTR of PTEN}

Forced overexpression of miR-625-3p deceased PTEN expression and increased $\mathrm{p}-\mathrm{AKT}$ expression in OVCAR3 (Fig. 6A) and OVCAR4 (Fig. 6B).

The dual-luciferase reporter assay demonstrated that luciferase activity did not change when mu-PTEN3'-UTR-pGL3 was co-transfected with miR-625-3p, while luciferase activity of Luc-PTEN was decreased when wt- PTEN-3'-UTR-pGL3 was co-transfected with miR-625-3p (Fig. 7).

\section{MiR-625-3p level on human ovarian high-grade serous cancer tissue}

Formalin-fixed paraffin-embedded (FFPE) tissue was obtained and qRT-PCR was carried out to examine miR-625-3p expression. We found that miR-572 expression was correlated with ovarian cancer stage (Table 2, Fig. 8A). In high-grade serous carcinoma expressing high-level miR-625-3p (Fig. 8B), Ki-67 proliferation index is increased. However, PTEN expression was decreased in high-grade serous carcinoma expressing high-level miR-625-3p. 


\section{Discussion}

Emerging evidence has demonstrated that miR-625-3p plays important roles in variety of different neoplasms. Fang et al showed that miR-625-3p improved growth, migration and invasion in thyroid papillary carcinoma by targeting astrocyte elevated gene 1 [14]. Rasmussen et al reported that expression of miR-625-3p was up-regulated in colorectal cancer cells and associated with resistance to oxaliplatin treatment and reduced overall survival [15]. Krischner and his co-workers found that miR-625-3p concentration is dramatically higher in plasma of patients with malignant pleural mesothelioma and demonstrated miR-625-3p could serve as a potential diagnostic marker [16]. Li et al showed that miR-625-3p decreased lymph node metastasis in gastric carcinoma by directly targeting enhancer of zeste homolog 2 [17]. Shapira et al reported that miR-625-3p concentration was elevated in plasma of patients with ovarian cancer and might serve as a biomarker to predict prognosis [18]. Our study showed that miR-625-3p promoted proliferation in ovarian high-grade serous carcinoma. These findings suggested that miR-625-3p plays important roles in high-grade ovarian serous carcinoma.

Cisplatin is used as a chemotherapy agent in variety of solid cancers including ovarian cancer. Resistance to cisplain-based chemotherapy is the major issue in patients with ovarian cancer. Different signaling pathways involve in development of cisplatin resistance including DNA damage repair, cell growth pathways and apoptotic pathways [19]. MicroRNAs have been demonstrated to involve in regulation of sensitivity of cisplatin in different neoplasms. For example, miR-34a could improve cisplatin sensitivity in non-small cell lung carcinoma [20] and miR-98-5p regulated Dicer1 axis and mediated cisplatin resistance in ovarian cancer [21]. We found miR625-3p overexpression improved growth of ovarian cancer cells with cisplatin treatment, however, low expression of miR-625-3p inhibited growth of ovarian cancer cells with cisplatin treatment. These findings suggested miR-625-3p regulated sensitivity of cisplatin in ovarian cancer cells. Furthermore, we showed miR$625-3 p$ regulated BAX and bcl-2 expression in the presentence of cisplatin. These results indicated miR625-3p might involve in cisplatin based chemotherapy resistance by regulating apoptosis pathway. We further showed miR-625-3p decreased PTEN expression in ovarian cancer cells by directly binding to 3'-UTR of PTEN. PTEN involves in development and progression in many neoplasms as tumor suppressor. Down-regulation of PTEN promotes cancer cell proliferation and inhibits apoptosis by regulating PI2K/AKT signaling.

Recent studies have shown that microRNAs involve in development of ovarian cancer by regulating PTEN signaling. Lou et al showed that miR-21 improved growth, migration and invasion in epithelial ovarian cancer cells by inhibiting PTEN expression [22]. Yang et al found that miR-214 and miR-150 were overexpressed in ovarian cancer cells and inhibited PTEN expression [23]. Li et al reported overexpression of miR-205 was associated with ovarian cancer progression by regulating both PTEN and SMAD4 signaling pathways [24]. Some studies also demonstrated that microRNAs involve in chemotherapy sensitivity in ovarian cancer by targeting PTEN signaling. Xiang et al reported miR-186 mediated cisplatin resistance in ovarian cancer cells by regulating PIK3R3 and PTEN signaling pathway [25]. Shi et al found miR-205-5p regulated cisplatin sensitivity by inhibiting PTEN expression in ovarian cancer cells [26].

In our study, PTEN expression was decreased in both ovarian cancer cell lines and human ovarian cancer specimen. These results indicated that miR-625-3p involved in ovarian proliferation via targeting PTEN signaling pathway.

In conclusion, our study suggested miR-625-3p might regulate growth and cisplatin based chemotherapy resistance in ovarian cancer by directly regulating PTEN signaling pathway. These findings indicate miR-625-3p could serve as a promising biomarker in ovarian cancer.

\begin{abstract}
Abbreviations
HGSOC: High-grade serous ovarian cancer; DMEM: Dulbecco's Modified Eagle's Medium; qRT-PCR: Quantitative Real-Time PCR; FFPE: Formalin-fixed paraffin-embedded.
\end{abstract}

Disclosure

No potential conflicts of interest were disclosed.

\section{Authors' contributions}

Lili Zhong and Xiumin Liu: performed experiments, write manuscript. Lixing Wang and Yu Liu: performed experiments, prepare figures and table. Duohan Zhang: Collected patient tissue, performed experiments. Yinlong Zhao: design the project, write manuscript and statistical analysis. All authors reviewed the manuscript. The author(s) read and approved the final manuscript.

\section{Funding}

This study was supported by special health project of Jilin Provincial Finance Department (3D518V613429), Special project of medical and Health Personnel in Jilin Province (2019SCZT026), Bethune Project (419161900018) and Special Health project of Jilin Province (2020SCZT061).

\section{Availability of data and materials}

The datasets used and/or analysed during the current study available from the corresponding author on reasonable request.

\section{Declarations}

Ethics approval and consent to participate

Our study was approved by Ethics Committee of Jilin University (approval No. JLU201908021135). All patients provided written informed consent prior to enrollment in the study. 


\section{Competing interests}

No potential conflicts of interest.

\section{Author details}

${ }^{1}$ The Second Hospital of Jilin University, Changchun 130041, China. ${ }^{2}$ Clinical Laboratory, Second Hospital of Jilin University, Changchun 130041, China. ${ }^{3}$ Department of Nuclear Medicine, The Second Hospital of Jilin University, No. 218, Ziqiang Street, Nanguan, Changchun 130041, Jilin, People's Republic of China.

Received: 16 June 2021 Accepted: 25 December 2021

Published online: 14 January 2022

\section{References}

1. Binju $M$, et al. Mechanisms underlying acquired platinum resistance in high grade serous ovarian cancer - a mini review. Biochim Biophys Acta Gen Subj. 2019;1863(2):371-8.

2. Chen $\mathrm{SN}$, et al. Microrna in ovarian cancer: biology, pathogenesis, and therapeutic opportunities. Int J Environ Res Public Health. 2019;16(9):1510.

3. Fang L, Kong D, Xu W. Microrna-625-3p promotes the proliferation, migration and invasion of thyroid cancer cells by up-regulating astrocyte elevated gene 1. Biomed Pharmacother. 2018;102:203-11.

4. Gadducci A, et al. Current strategies for the targeted treatment of highgrade serous epithelial ovarian cancer and relevance of brca mutational status. J Ovarian Res. 2019;12(1):9.

5. Hayes J, Peruzzi PP, Lawler S. micrornas in cancer: biomarkers, functions and therapy. Trends Mol Med. 2014;20(8):460-9.

6. Kirschner MB, et al. Increased circulating Mir-625-3p: a potential biomarker for patients with malignant pleural mesothelioma. J Thorac Oncol. 2012;7(7):1184-91.

7. Li J, et al. Upregulation Of Mir-205 transcriptionally suppresses Smad4 and pten and contributes to human ovarian cancer progression. Sci Rep. 2017;7:41330

8. LiY, et al. Microrna-625-3p inhibits gastric cancer metastasis through modulating Ezh2. Eur Rev Med Pharmacol Sci. 2020;24(3):1177-85.

9. Lou Y, et al. Microrna-21 promotes the cell proliferation, invasion and migration abilities in ovarian epithelial carcinomas through inhibiting the expression of pten protein. Int J Mol Med. 2010;26(6):819-27.

10. Matulonis UA. Management of newly diagnosed or recurrent ovarian cancer. Clin Adv Hematol Oncol. 2018;16(6):426-37.

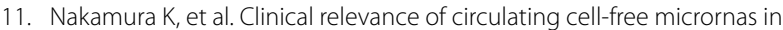
ovarian cancer. Mol Cancer. 2016;15(1):48.

12. Rao G, et al. Microrna-195 controls micu1 expression and tumor growth in ovarian cancer. Embo Rep. 2020;21(10):E48483.

13. Rasmussen $\mathrm{MH}$, et al. High expression of microrna-625-3p is associated with poor response to first-line oxaliplatin based treatment of metastatic colorectal cancer. Mol Oncol. 2013;7(3):637-46.

14. Rocha CRR, et al. Dna repair pathways and cisplatin resistance: an intimate relationship. Clinics. 2018;73(Suppl 1):E478s

15. Shapira I, et al. Circulating biomarkers for detection of ovarian cancer and predicting cancer outcomes. Br J Cancer. 2014;110(4):976-83.

16. Shi X, et al. Mir-205-5p mediated downregulation of pten contributes to cisplatin resistance in C13k human ovarian cancer cells. Front Genet. 2018;9:555

17. Si W, et al. The role and mechanisms of action of micrornas in cancer drug resistance. Clin Epigenetics. 2019;11(1):25.

18. Song C, et al. Mir-34a sensitizes lung cancer cells to cisplatin via P53/Mir34a/Mycn axis. Biochem Biophys Res Commun. 2017:482(1):22-7.

19. Vang R, Shih IM, Kurman RJ. Ovarian low-grade and high-grade serous carcinoma: pathogenesis, clinicopathologic and molecular biologic features, and diagnostic problems. Adv Anat Pathol. 2009;16(5):267-82.

20. Wang $X$, et al. Circulating micrornas as novel potential diagnostic biomarkers for ovarian cancer: a systematic review and updated metaanalysis. J Ovarian Res. 2019;12(1):24.

21. Wang Y, et al. Mir-98-5p contributes to cisplatin resistance in epithelial ovarian cancer by suppressing mir-152 biogenesis via targeting dicer 1. Cell Death Dis. 2018;9(5):447.
22. Wu AY, et al. Fn14 overcomes cisplatin resistance of high-grade serous ovarian cancer by promoting Mdm2-mediated P53-R248q ubiquitination and degradation. J Exp Clin Cancer Res. 2019;38(1):176.

23. Xiang Y, et al. Mir-186 bidirectionally regulates cisplatin sensitivity of ovarian cancer cells via suppressing targets Pik3r3 and pten and upregulating apaf1 expression. J Cancer. 2020;11(12):3446-53.

24. Xing F, Wang S, Zhou J. The expression of microrna-598 inhibits ovarian cancer cell proliferation and metastasis by targeting uri. Mol Ther Oncolytics. 2019;12:9-15.

25. Yang B, Sun L, Liang L. Mirna-802 suppresses proliferation and migration of epithelial ovarian cancer cells by targeting Ywhaz. J Ovarian Res. 2019:12(1):100

26. Yang $\mathrm{H}$, et al. Microrna expression profiling in human ovarian cancer: mir-214 induces cell survival and cisplatin resistance by targeting Pten. Cancer Res. 2008;68(2):425-33.

\section{Publisher's Note}

Springer Nature remains neutral with regard to jurisdictional claims in published maps and institutional affiliations.
Ready to submit your research? Choose BMC and benefit from:

- fast, convenient online submission

- thorough peer review by experienced researchers in your field

- rapid publication on acceptance

- support for research data, including large and complex data types

- gold Open Access which fosters wider collaboration and increased citations

- maximum visibility for your research: over $100 \mathrm{M}$ website views per year

At $\mathrm{BMC}$, research is always in progress.

Learn more biomedcentral.com/submissions 